Review

\title{
Pathogenic Role of Exosomes in Epstein-Barr Virus (EBV)-Associated Cancers
}

\author{
Sin-Yeang Teow ${ }^{1 凶}$, Kitson Liew ${ }^{2}$, Alan Soo-Beng Khoo ${ }^{2,3}$, Suat-Cheng Peh ${ }^{1,4}$ \\ 1. Sunway Institute for Healthcare Development (SIHD), Sunway University, Jalan Universiti, Bandar Sunway, 47500 Subang Jaya, Selangor Darul Ehsan, \\ Malaysia. \\ 2. Molecular Pathology Unit, Cancer Research Centre (CaRC), Institute for Medical Research (IMR), Jalan Pahang, 50588 Kuala Lumpur, Malaysia. \\ 3. Institute for Research, Development and Innovation, International Medical University (IMU), Jalan Jalil Perkasa 19, Bukit Jalil, 57000 Kuala Lumpur, \\ Malaysia. \\ 4. Anatomical Pathology Department, Sunway Medical Centre, Jalan Lagoon Selatan, Bandar Sunway, 47500 Subang Jaya, Selangor Darul Ehsan, Malaysia.
}

$\square$ Corresponding author: Sin-Yeang Teow, PhD., Research fellow, Sunway Institute for Healthcare Development (SIHD), Sunway University, Jalan Universiti, Bandar Sunway, 47500 Subang Jaya, Selangor Darul Ehsan, Malaysia; Email: ronaldt@sunway.edu.my; Alternate email: ronaldsyeang@gmail.com; Tel : +6 0374918622 (Ext. 7369); Mobile: +6 0165982292

( ) Ivyspring International Publisher. This is an open access article distributed under the terms of the Creative Commons Attribution (CC BY-NC) license (https://creativecommons.org/licenses/by-nc/4.0/). See http://ivyspring.com/terms for full terms and conditions.

Received: 2017.02.07; Accepted: 2017.06.27; Published: 2017.09.21

\begin{abstract}
Exosomes are 40- to 100-nm membrane-bound small vesicles that carry a great variety of cellular cargoes including proteins, DNA, messenger RNAs (mRNAs), and microRNAs (miRNAs). These nanovesicles are detected in various biological fluids such as serum, urine, saliva, and seminal fluids. Exosomes serve as key mediators in intercellular communication by facilitating the transfer and exchange of cellular components from cells to cells. They contain various pathogenic factors whereby their adverse effects have been implicated in multiple viral infections and cancers. Interestingly, accumulating evidences showed that exosomes derived from tumour viruses or oncoviruses, exacerbate virus-associated cancers by remodelling the tumour microenvironment. In this review, we summarize the contributing factors of Epstein-Barr virus (EBV) products-containing exosomes in viral pathogenesis and their potential implications in EBV-driven malignancies. Understanding the biological role of these exosomes in the disease would undoubtedly boost the development of a more comprehensive strategy to combat EBV-associated cancers and to better predict the therapeutic outcomes. Furthermore, we also highlight the potentials and challenges of EBV products-containing exosomes being employed as diagnostic markers and therapeutic targets for EBV-related cancers. Since these aspects are rather underexplored, we attempt to underline interesting areas that warrant further investigations in the future.
\end{abstract}

Key words: Exosome; Epstein-Barr virus; EBV-associated cancer; LMP; nasopharyngeal carcinoma

\section{Introduction}

The current progress of therapy or therapeutics development against cancers is still not able to address the elevating cancer-related morbidity and mortality worldwide. Multiple challenges continue to exist in the development of anticancer drugs, which include the lack of suitable study models [1], as well as conundrums over drug toxicity [2], delivery [3], and bioavailability [4]. Accumulating evidences demonstrate that surrounding stromal cells such as tumour-infiltrating lymphocytes, fibroblasts, mesenchymal stem cells and macrophages/ monocytes in tumour regions may contribute to the failure of anticancer drug treatment $[5,6]$. Other pathogenic factors derived from tumour viruses (also known as oncoviruses) $[7,8]$ and tumour-derived exosomes $[9,10,11]$ may also help to establish a protumoural microenvironment by orchestrating multiple tumour-mediated signalling pathways hence complicating the anticancer treatment.

Exosomes are small membrane bound vesicles that are secreted by cells. These extracellular vesicles (EVs) carry a wide range of molecules that have 
implications in cell-to-cell communications, hence contributing to pathogenesis of various diseases and infections $[9,10,11,12,13]$. There are several types of EVs which are apoptotic bodies (500-2000nm), microvesicles (50-1000nm), and exosomes (30-100nm) which are usually differentiated by cellular origins but not size [14,15]. Due to the lack of distinctive vesicle-specific marker within the membrane-bound small vesicles, the term exosomes are often used interchangeably with small EVs [16]. Exosomes are defined as membrane-bound vesicles formed within multivesicular bodies (MVBs) or late endosomes which secreted from the cell [15]. Exosomes are formed through an endocytic pathway (Figure 1), endocytosed materials are delivered to early endosomes and they are either sent for degradation after fusion with lysosomes, or enter the secretory pathway via MVBs. The vesicles within MVBs are known as intraluminal vesicles (ILVs) and they are called exosomes following their release from the MVB upon fusion with plasma membrane to the milieu (Figure 1) [14,15].

The roles of exosomes in viral pathogenesis have been recently reviewed, including those in human immunodeficiency virus type I (HIV-I) [12,13], EBV $[17,18]$, hepatitis $\mathrm{C}$ virus (HCV) $[19,20]$, and human T-cell lymphotropic virus type I (HTLV-1) [21,22]. More importantly, some of these exosomes have been shown to aggravate the tumour development and dissemination [14,23]. For examples, exosomes from EBV-infected nasopharyngeal carcinoma (NPC) cells contain hypoxia-inducible factor-1a (HIF1a) and latent membrane protein-1 (LMP-1) that contribute to the tumour development and progression in NPC $[23,24]$. NPC-derived exosomes also play multiple roles in immune evasion in order to support and sustain the tumour growth $[24,25]$. On the other hand, the human papilloma virus (HPV)-associated exosomes have been shown to contain microRNAs that were associated with cervical cancer [26].

More than $90 \%$ of world's population is infected with EBV and its contributions to various lymphoand epithelial-malignancies such as NPC, gastric carcinoma (GC), Burkitt lymphoma (BL), Hodgkin lymphoma (HL), and Non-Hodgkin lymphoma (NHL) are well-documented [27,28]. Exosomes have been shown to play important roles in the EBV infection and spreading $[17,18]$. However, little is known about the functions of EBV product-containing exosomes in the cancer development and progression that have been partly discussed in several reviews that focus on virus-derived exosomes $[14,22,29,30]$. In this review, we summarize the functions of exosomes in EBV pathogenesis and their contributions to several EBV-driven malignancies based on current findings, particularly on NPC. We also discuss the potentials of developing the anti-cancer therapy that target the EBV-associated exosomes and exosome-based biomarkers for diagnosis of EBV-related cancers. Moreover, we attempt to highlight the entailed challenges and limitations in the aforementioned endeavours.

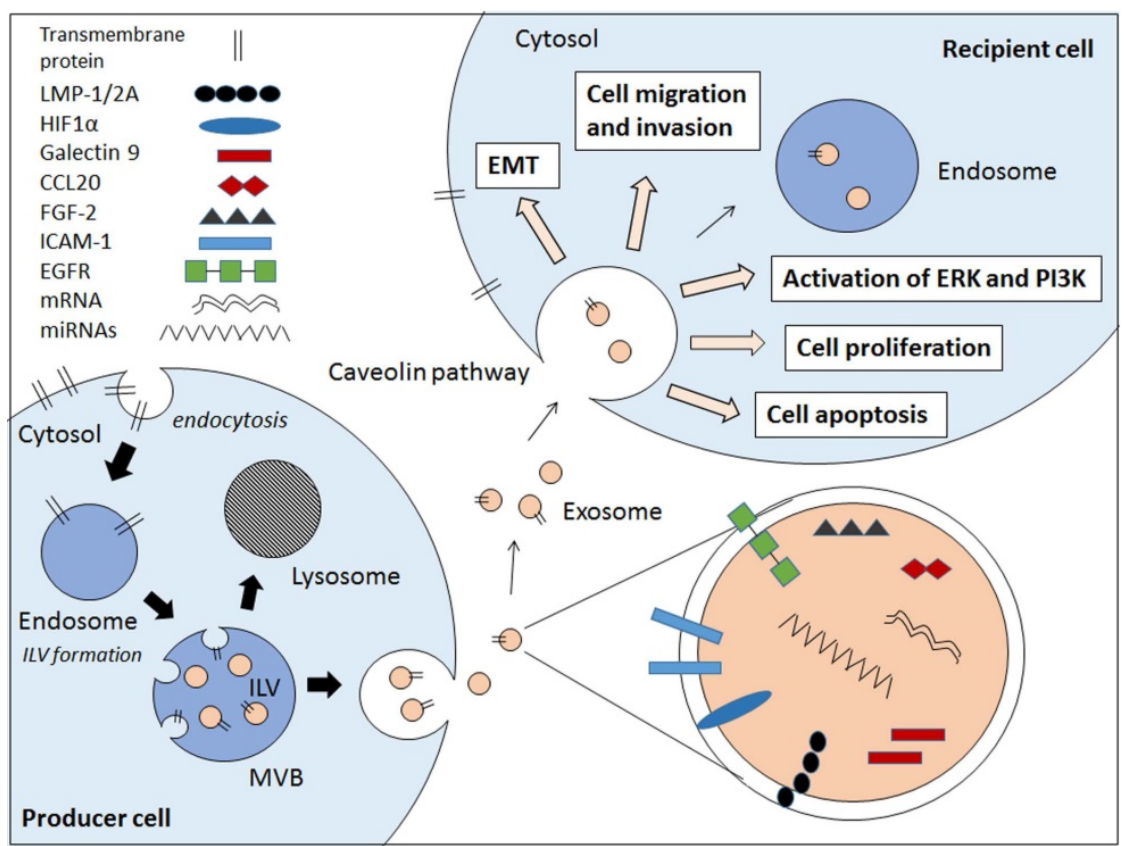

Figure 1. Schematic diagram showing the EBV-related pathogenic factors that contributes to cancer pathogenesis via exosomal pathway. Internal vesicles or multivesicular bodies (MVBs) are formed by the inward budding of cellular compartments. The cargo is packaged into intraluminal vesicle (ILVs) within MVBs. The MVBs contain a wide range of pathogenic factors depending on the producer cell. The MVBs either fuse with lysosomes to degrade MVB contents or fuse with cell membrane to release exosomes. The exosomes enter the recipient cell via caveolae-mediated endocytosis and the pathogenic contents are implicated in the EBV-associated cancer pathogenesis. 


\section{Exosomes in EBV infection and persistence}

Exosomes are known to transfer proteins, DNAs, mRNAs, and miRNAs from cells to cells to modulate diverse cellular processes [14,22]. In recent years, works on pathogenic role of oncogenic virus-derived exosomes have been mostly focused on the cancer pathogenesis [29,31]. In the case of EBV-associated exosomes, their role in EBV infection and persistence is surprisingly less understood. It is crucial to understand the biology of exosomes in the virus pathogenesis given that virus-derived exosomes are likely to contain various virus-related proteins/genes that modulate the viral infection which may in turn, contribute to the cancer development and/or progression later on. Of note, the exosome contents largely vary depending on the state of viral infection. There is a general notion that the EBV infection aggravates the local infection site, hence increasing the frequency of the occurrence of EBV-related malignancies [27,32,33]. As EBV-associated exosomes are majorly detected in the virally infected cells, they may possibly aid EBV in manipulating the surrounding tumour microenvironment to favour tumour growth and survival. This can be supported by the work of Ahmed et al. who reported that EBV could hijack the exosomal pathway for the purposes of virus egress and immune evasion [34]. Cumulative evidences also showed that EBV-associated exosomes specifically pack a variety of viral components (eg., LMP-1, LMP-2A, EBERs, viral RNAs, miRNAs, etc) which may promote the EBV infection $[14,22,23,24,25,31]$. The following subsection shall discuss the role of exosomes containing different EBV products in contributing to EBV infection and persistence.

\section{Role of latent membrane proteins (LMPs)-containing exosomes}

EBV latent infection, in general can be divided into three types (type I, II, and III). Latency type I is characterized by a high expression of Epstein-Barr virus nuclear antigen-1 (EBNA-1) which is usually seen in BL and GC. On the other hand, EBNA-1, LMP-1, and LMP-2 expressions are commonly seen in latency type II which occurs in the course of HL and NPC. In the case of latency type III, all latent proteins/antigens are expressed as commonly seen in acquired immune deficiency syndrome (AIDS)-related lymphomas and lymphoblastoid cell lines (LCLs) [27,32].

EBV LMP-1 and LMP-2 have profound effects on both virus and cancer pathogenesis. LMP-1 is the major viral oncogene expressed in most
EBV-associated cancers, which mainly fall under the categories of latency type II and III as mentioned above. LMP-1 can be detected in exosomes from EBV-infected cancer cells and is transferable from producer to recipient cells using exosomes as vehicles $[23,31]$. This oncoprotein may not be directly involved in the establishment of EBV infection, but it contributes significantly to the success of virus spreading by ensuring the high production of infectious virions [35]. Previously, immunohistochemical study on NPC tumour tissues have first demonstrated the positive correlation between protein levels of LMP-1 and exosomal marker CD63, suggesting that LMP-1 up-regulates the exosomal secretion during NPC progression [23]. Indeed, recent findings by Hurwitz et al. showed that both exosomal proteins' level and extracellular vesicle secretion increased following LMP-1 expression. This resulted in greater LMP-1 accumulation and packaging in CD63-positive exosomes which can be impaired upon CD63 ablation [36]. Given the above considerations, it is likely that LMP-1 could also enhance EBV spreading by stimulating the production of pathogenic exosomes which include the exosomal LMP-1 as well.

Work by Nanbo et al. on the other hand, showed that LMP-1-containing exosomes increased the expression of intercellular adhesion molecule 1 (ICAM-1) that subsequently increased the proliferation rate of type III latency EBV-infected cells [37]. In contrast, exosomal LMP-1 derived from LCLs have been shown to inhibit the proliferation of peripheral blood mononuclear cells (PBMCs) [38]. Similarly, NPC-released exosomes containing LMP-1 and galectin 9 also exhibit the intrinsic T-cell inhibitory activity [24]. These findings collectively suggest that exosomes containing LMP-1 can promote EBV pathogenesis mainly by down-regulating the antiviral immune responses whilst sustaining and supporting the growth of EBV-infected cells.

Similar to LMP-1, presence of LMP-2A has also been detected in exosomes collected from EBV-infected LCLs [39]. Interestingly, it has been shown that cholesterol depletion via methyl-beta-cyclodextrin (MCD) treatment can elevate the exosomal LMP-2A level and stability [40]. However, the virus-specific effect of the exosome-derived LMP-2A in the EBV pathogenesis has not been evaluated before. Hence, little is known for the role of exosomal LMP-2A in virus pathogenesis as compared to the exosomal LMP-1. This question warrants future investigations as the pathogenic effects of cellular LMP-2A have been profoundly implicated in EBV infection [41, 42] and such effects could also be potentially manifested by 
the exosome-derived LMP-2A.

\section{Role of EBV RNAs}

In addition to LMPs, EBV-associated exosomes could also contain the Epstein-Barr virus-encoded small RNAs (EBERs). EBERs (i.e., EBER-1 and EBER-2) are non-coding RNAs that are abundantly expressed through the exosomal secretion in EBV-infected cells $[34,43]$. These EBERs are mainly responsible for the cellular transformation and anti-viral innate immunity [44]. Ahmed and co-workers demonstrated that EBERs were excreted in the form of exosomes along with lupus antigen (La) ribonucleoprotein, which is an EBER-binding protein [34]. However, the pathogenic effects of EBER-carrying exosomes upon their internalization into the neighbouring cells remain unexplored, which requires further investigation.

EBV exosomes may also contain other viral mRNAs and miRNAs that are known to mediate EBV pathogenesis and persistence $[18,45,46]$. For instances, exosomes derived from EBV-transformed LCL can transfer the mature EBV-encoded miRNAs to the recipient dendritic cells (DC) to suppress the expression of EBV target genes in the cells [45]. While the EBV-containing exosomes play significant roles in the viral pathogenesis, the mechanism mediating the internalization of these exosomes into the target cells is still unclear although it underlies the phenotypic modulation of target cells by the exosomes. Nanbo and co-worker demonstrated that the exosomes from EBV-uninfected, type I and type III latency EBV-infected cells internalized into the EBV-uninfected epithelial target cells via caveola-dependent endocytosis [37]. Since EBV products-containing exosomes could bind to various proteins/ receptors on target cells such as CD21 on B cells [47], exosome internalization is anticipated to be mediated via receptor-associated pathways. More studies are still required to decipher the exosome internalization pathway that facilitates EBV pathogenesis and persistence, which may contribute to EBV-associated carcinogenesis.

\section{EBV-Associated Exosomes in Tumorige- nesis and Tumour Dissemination}

Tumour-derived exosomes have been known to aggravate the progression and invasion of various cancers including breast, ovarian, and brain cancers $[14,22]$. While the EBV-associated exosomes facilitate the EBV infection and spreading [35], there are evidences showing that these exosomes contribute to various EBV-associated cancers such as NPC, GC, and BL [23]. For instances, EBV oncoproteins LMP-1 released from exosomes has been involved in the activation of several tumour-related signalling pathways (i.e., EGFR/PI3K and AKT/ERK), and consequently promote tumour development and progression [31]. In this section, we discuss the pathogenic role of EBV product-containing exosomes in various EBV-associated lymphoid and epithelial malignancies. Recent reports that show the exosomes-driven tumorigenesis and progression in the EBV-related cancers are summarized in Table 1.

\section{Exosomal LMPs in EBV-related cancers}

LMP-1 is expressed in EBV-associated cancers that display latency type II and III which include NPC, HL, and AIDS-related lymphoma. Exosome-derived LMP-1 mainly attenuates the immune response during the establishment of EBV infection in the target cells. The immunosuppressive roles of exosomal LMP-1 are evident as shown in several studies on EBV-associated cancers. LMP-1-containing exosomes derived from EBV-infected lymphoblastoid and NPC cells were found to inhibit the T-cell activation and proliferation via a mechanism that could be mediated by the critical inhibitory motif in the conserved transmembrane domain of LMP-1 [24,48]. Flanagan and co-workers also proposed that exosomes containing LMP-1 may block the activity of infiltrating T-cells upon uptake of these exosomes and this blockade may contribute to the immune evasion of tumour cells [38]. In another study, Ceccarelli and colleagues showed that the LMP-induced the expression and extracellular release via exosomes of fibroblasts growth factor 2 (FGF-2), a proangiogenic factor in tumour invasion. More remarkably, the authors also showed that LMP-1 could accumulate and concentrate together with FGF-2 in multivesicular bodies prior to their exosomal release via the $\mathrm{Na}(+) / \mathrm{K}(+)$-ATPase-dependent pathway [49].

Meckes and co-workers on the other hand, showed that LMP-1-containing exosomes from LMP-1-overexpressed C666-1 NPC cells contained EGFR and PI3K as well. These exosomes were able to transfer LMP-1 and activate the ERK and AKT signalling pathways in multiple cell types, including fibroblast, uninfected epithelial, and HUVEC cells [31]. This finding suggests that the transfer of oncogenic LMP-1 may modulate the behaviour of surrounding stromal cells, causing their aberrant activation that favours primary tumour growth via enhanced vascularization as in the case example of HUVEC cells. It can be envisaged that neighbouring epithelial cells could be possibly transformed into cancer cells as a consequence of the oncogenic LMP-1 transfer via the exosomal pathway. Apart from that, exosomal LMP-1 also up-regulated the expression of 
ICAM-1 or CD54 [37] in which their elevated expressions have been found in various cancers including NPC [50], GC [51], and NHL [52]. Interestingly, Verweij et al have shown that LMP-1 can escape lysosomal degradation by first accumulating into endosomes, followed by exosomal secretion mediated by its interaction with the exosomal marker CD63 [53]. High correlation of LMP-1 and CD63 also suggested that the LMP-1 up-regulated the exosomal secretion which may promote the EBV-associated cancers. [23]. These observations may help to shed light on its constitutive oncogenic activities in various EBV-associated cancers [54].

Cumulative findings suggest that the exosomal LMP-1 may promote the growth and progression of EBV-associated cancers (Table 1). Numerous studies have shown that cellular LMP-1 play multiple roles in the EBV latent infection and tumorigenesis particularly via activation of c-Jun $\mathrm{N}$ terminal kinase (JNK) [55], NF-kappaB [56], p38[57], phosphatidylinositol 3 kinase (PI3K)/Akt [58], MAPK [59], and JAK/STAT [60] signalling. Our group also has reported that LMP-1 overexpression promoted the degradation of p53 tumour suppressor protein which intimates that $\mathrm{p} 53$ could be inactivated in NPC by LMP-1 despite being found to be rarely mutated in the disease [61,62]. It remains to be seen whether exosome-derived LMP-1 retains all the functions of the cell-derived LMP-1. Similarly, epidermal growth factor receptor (EGFR) which is a LMP-1-inducible tumour-promoting target protein [63], is also secreted by exosomes [31]. Following the exosomal delivery, the exosomal EGFR may contribute to the cancer pathogenesis. Notwithstanding all the above findings, the exact mechanisms of the LMP-1's exosomal packaging, trafficking, internalization, and secretion are still not fully understood and these require future investigation.

As for LMP-2A, it has been shown to integrate into the exosomes and is released into the recipient cells. Yet, the role of exosomal LMP-2A in carcinogenesis is underexplored despite the fact that cell-associated LMP-2A have been extensively studied for carcinogenesis $[64,65,66,67,68]$. The fact that the LMP-2A is expressed consistently in all EBV-associated NPCs as compared to the restrictive expression of LMP-1 may suggest the clinical significance of exosomal LMP2A [69]. Further investigation is warranted to examine the function of exosomal LMP-2A in the cancer development and progression.

Table 1. Evidences supporting the role of EBV-associated exosomes in tumour progression.

\begin{tabular}{|c|c|c|c|c|c|}
\hline Exosomal proteins & Producer cell & Recipient cell & Cancer type & Pathogenic or oncogenic effect of EBV-exosomes & Reference \\
\hline $\begin{array}{l}\text { LMP-1 and } \\
\text { HIF1a }\end{array}$ & $\begin{array}{l}\text { LMP-1-NP69 and } \\
\text { LMP--AdAH NPC cell } \\
\text { lines }\end{array}$ & $\begin{array}{l}\text { NP69 and } \\
\text { AdAH }\end{array}$ & NPC & $\begin{array}{l}\text {-LMP-1 induces HIF1a expression } \\
\text {-Induction of epithelial-mesenchymal transition } \\
\text { (EMT) } \\
\text {-Increase of cell migration and invasiveness }\end{array}$ & 23 \\
\hline $\begin{array}{l}\text { LMP-1 and } \\
\text { Galectin } 9\end{array}$ & $\begin{array}{l}\text { C15 and C17 NPC } \\
\text { xenograft tumour lines }\end{array}$ & PBMC-derived T-cells & NPC & -Inhibition of peripheral blood T-cell proliferation & 24 \\
\hline $\begin{array}{l}\text { LMP-1 and } \\
\text { Galectin } 9\end{array}$ & $\begin{array}{l}\mathrm{C} 15, \mathrm{C} 17, \mathrm{C} 666-1 \mathrm{NPC} \\
\text { xenograft tumour lines } \\
\text { and NPC patients }\end{array}$ & $\begin{array}{l}\text { PBMC-derived } \\
\text { EBV-specific CD4 } \\
\text { T-cell clones }\end{array}$ & NPC & -Induction of EBV-specific CD4+ T-cell apoptosis & 86 \\
\hline CCL20 & $\begin{array}{l}\mathrm{C} 15 \text { and } \mathrm{C} 17 \mathrm{NPC} \\
\text { xenograft tumour lines }\end{array}$ & PBMC-derived T-cells & NPC & $\begin{array}{l}\text {-CCL20 facilitated human Treg recruitment, } \\
\text { expansion, and upregulated their suppressive } \\
\text { functions } \\
\text {-Promote conversion of CD } 4+C D 25-T \text { cells to Treg }\end{array}$ & 25 \\
\hline LMP-1 and FGF-2 & $\begin{array}{l}\text { LMP-1/FGF2-AdAH } \\
\text { NPC cell line }\end{array}$ & HUVEC & NPC & $\begin{array}{l}\text {-LMP-1 induces expression of angiogenic factor } \\
\text { FGF-2 and exosomal secretion } \\
\text {-Increase of cell proliferation in HUVEC }\end{array}$ & 49 \\
\hline $\begin{array}{l}\text { LMP-1 and } \\
\text { ICAM-1 }\end{array}$ & $\begin{array}{l}\text { Mutu-, Mutu I, Mutu III } \\
\text { BL cell lines and LCL }\end{array}$ & $\begin{array}{l}\text { CNE-1, HONE-1, } \\
\text { NU-GC-3, A549 }\end{array}$ & $\begin{array}{l}\text { BL, NPC, GC, } \\
\text { lung }\end{array}$ & $\begin{array}{l}\text {-LMP-1 induces expression of ICAM-1 } \\
\text {-Increase of cell proliferation in CNE-1 NPC cell line }\end{array}$ & 37 \\
\hline $\begin{array}{l}\text { LMP-1, EGFR and } \\
\text { miRNAs }\end{array}$ & $\begin{array}{l}\text { C666, LMP-1-C666, } \\
\text { C33A cell lines and C15 } \\
\text { NPC xenograft tumour } \\
\text { line }\end{array}$ & HUVEC, C33A, Rat-1 & NPC & $\begin{array}{l}\text {-Transfer of BART miRNAs to HUVEC cells } \\
\text {-LMP-1 induces expression of EGFR } \\
\text {-Activation of ERK and PI3K/Akt signalling } \\
\text { pathways }\end{array}$ & 31 \\
\hline BHRF1-3 miRNAs & LCL & MoDC and HeLa & Not specific & $\begin{array}{l}\text {-EBV BART and BHRF1-3 miRNAs are transferred } \\
\text { between B-cell and non-B cells } \\
\text {-EBV BHRF1-3 miRNAs suppress CXCL11 } \\
\text { expression }\end{array}$ & 45 \\
\hline
\end{tabular}




\section{Exosomal RNAs in EBV-related cancers}

miRNAs are small non-coding RNAs with size of about 20 nucleotides that regulate various cellular processes $[70,71]$. The mature miRNAs interact with the target mRNAs and block their activities by repressing the translation. The effects of miRNAs have been implicated in EBV-associated cancers such as NPC $[72,73,74]$, GC $[68,75,76]$, and BL $[77,78]$. Pandya and colleagues have in fact shown that viral miRNA expression is consistently higher in cancerous tissues as compared to the adjacent non-cancerous counterparts in various solid malignancies [79]. It was also shown that the expression of these viral miRNAs is linked to significantly worse clinical outcome among early stage cancer patients, which potentially indicates the potential devastating pathogenic roles of viral miRNAs in cancer [79]. The tumour-promoting miRNAs are also detected in exosomes. Due to the exosome biogenesis, it is thought that the biological effects of exosome-derived miRNAs may be slightly different compared to the cell-derived miRNAs. However, cumulative findings suggest that exosomal miRNAs retains their tumorigenic effects in various cancers similar to the cell-derived miRNAs $[80,81,82,83,84]$. For instance, Melo and colleagues demonstrated that breast cancer cells secreted exosomes containing RNA induced silencing complex (RISC) proteins, Dicer, TAR RNA binding protein (TRBP), and Argonaute 2 (AGO2), and these exosomes are capable of processing the precursor miRNAs into the active miRNAs that induce tumour formation [80]. Interestingly, Ye and co-workers also demonstrated that exosomal miRNAs promoted the tumour progression in NPC [82]. However, the profile of miRNA-containing exosomes and the pathogenesis mechanism of the NPC-derived exosomes were not evaluated in this study. On the other hand, Choi and co-workers showed that miR-BART15-3p could be detected in the EBV-associated exosomes and its expression level was 2 to 16 -fold higher in the exosomes compared to the cellular level [85], but its roles in tumorigenesis was again, not studied. Overall, current findings suggest that EBV miRNAs may have high tumorigenic activities but more studies need to be carried out to support this speculation.

Similarly, EBV-associated exosomes have been shown to carry mRNAs encoding for EBV oncogenes such as LMP-1, LMP-2, EBNA-1, and EBNA-2 and small RNA EBERs [18,34] (Figure 1). In regards to cellular EBERs, our group has previously demonstrated that NPC cells stably expressing EBER-1 and -2 consistently displayed an increased growth rate [86]. Despite the potential oncogenic properties of exosomal EBV-encoded mRNAs, whether or not these mRNAs are transferable to recipient cells and exert the tumorigenic effects in EBV-associated cancers remains to be shown.

\section{Other exosomal proteins in EBV-related cancers}

In addition to LMPs, EBERs, EBV-related miRNAs and mRNAs, exosomes containing other pathogenic proteins have also been reported to promote EBV-related cancers such as the transcription factor Galectin-9 [24,87], Deoxyuridine triphosphatase (dUTPase) [88], EGFR [31,89], HIF1a [23], and other interleukins [90] (Figure 1). Keryer-Bibens and colleagues demonstrated that the EBV-infected NPC-derived xenograft cells, C15 and C17 released the galectin-9-containing exosomes which have inhibitory action against PBMC-derived $\mathrm{T}$ cell proliferation [24]. Similarly, high expression of exosomal galectin-9 was also detected in plasma from NPC patients and NPC-tumour xenografted mice and these exosomes induced apoptosis in the EBV-specific CD4+ T cells in the galectin-9-dependent manner [87]. In the case of dUTPase, an increased amount of this protein has been detected in exosomes derived from the EBV-positive BL cell line, Raji which are found to induce the cytokine release from DCs and PBMCs which may activate the NF-kappaB pathway [88]. Interestingly, EBV-associated exosomes also carry hypoxia-inducible factor-1a (HIF1a), a pro-tumorigenic molecule [23]. Aga and coworkers have shown that LMP-1-inducible exosomal HIF1a contributed to the epithelial-mesenchymal transition (EMT) that may suggest its pro-metastatic role in promoting cancer progression. As one of the important therapeutic target for anti-cancer therapy, EGFRs are also over-expressed in NPC-derived exosomes [31]. The same group have shown that the exosomal EGFR expression correlates with the LMP-1 level and could be induced by LMP-1. More importantly, the exosomes derived from the Raji cells and other EBV-infected cell lines are enriched with various immune modulators such as interferon-inducible protein 16 (IFI16), cleaved caspase-1, IL-1 $\beta$, IL-18, and IL-33 [90]. The presence of these proteins in the exosomes may suggest that the EBV utilize the host exosomal pathway in immune escape hence contributing to the progression and maintenance EBV-associated cancers.

\section{Targeting EBV-associated Exosomes for Diagnostics and Anti-Cancer Therapy}

As described in earlier sections, cumulative findings have shown the marked tumorigenic role of exosomes in EBV-associated cancers. This suggests that these exosomes potentially represent useful 
biomarkers and strategies targeting these exosomes may be developed as a potent anti-cancer therapy. Similarly, EBV-associated exosomes that contain several other pathogenic proteins can also be targeted for cancer biomarker and therapy development (Figure 2). In this section, we summarize findings from the current works exploiting the EBV-associated exosomes as diagnostic markers as well as for the development of anti-cancer therapy. We also attempt to highlight the potential challenges and limitations that may be encountered in the above efforts.

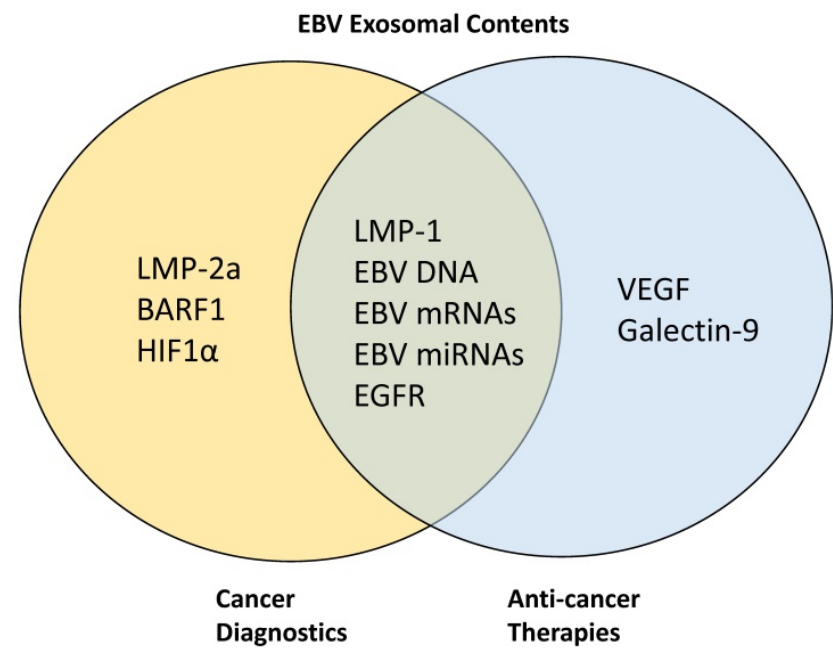

Figure 2. Target proteins in EBV-associated exosomes for cancer diagnostics and anti-cancer therapies. LMP-2A, BARFI, and HIFl $\alpha$ have been previously targeted for cancer diagnostics while VEGF and galectin- 9 are targeted for anti-cancer therapy development. Other proteins such as LMP-1, EGFR, EBV DNA, mRNAs, and miRNAs are being targeted simultaneously for both diagnostics and therapeutic purposes.

\section{EBV-associated exosomes as cancer diagnostic marker}

As abovementioned, exosomes containing EBV-related or other pathogenic factors may contribute to the cancer development and progression. These factors can potentially serve as useful markers for diagnosis or prognosis in cancers, particularly in the EBV-associated cancers. Recent advances in methods have facilitated the isolation of exosomes from the biofluids, making it a more feasible approach for the development of non-invasive liquid biopsy-based diagnostics [27,91]. The potential of developing exosomes into the biomarkers for cancers have been recently reviewed $[92,93,94,95]$. There have also been evidences suggesting that the EBV-associated oncoproteins can be targeted for cancer diagnosis. For instance, Houali and colleagues showed that both EBV oncoproteins LMP-1 and BARF1 could be detected in serum and saliva of NPC patients, and the secreted LMP-1 were strongly associated with the exosome-like vesicles
[96]. The fact that the exosomes are abundantly expressed in patient serum and saliva [12] further support the potential of targeting the oncoprotein-enriched exosomes in cancer diagnosis. Mao and coworkers also highlighted the potential of LMP-1 and LMP-2A as the candidate biomarkers for diagnosis and prognosis of patients with NK/T-cell lymphoma [97]. In addition to EBV-related proteins, other pathogenic factors that are enriched in exosomes as abovementioned such as EBV DNA [98,99], EBV mRNAs [100,101], EBV miRNAs $[102,103]$, Galectin-9 $[87,104]$ have also been shown to be potential biomarkers for cancer diagnosis. On the other hands, the diagnostic value of EGFR and HIF1a has been previously demonstrated in other cancer types [105, 106]. More poignantly, exosomes have been shown to protect their cargoes from degradation. For instance, the encapsulated mRNAs and miRNAs are being protected from RNAses [107]. The stability of the exosomal contents therefore, makes them the attractive biomarker candidates for cancer diagnosis. Further investigations are needed to evaluate the sensitivity and specificity of exosome-containing pathogenic proteins to be used as potential biomarkers for cancer diagnosis particularly in EBV-associated cancers.

\section{EBV-associated exosomes as anticancer target}

It has been reported that the tumour-derived exosomes could be the one of the major reasons for the treatment failure in cancers [108,109]. In this regard, cancer progression could be inhibited by blocking the activities of these exosomes. Figure 1 summarizes the pathogenic and tumorigenic factors derived from the EBV-associated exosomes and the potential targets for anti-cancer therapy development. Indeed, EBV-related proteins and nucleic acids (eg., DNA, mRNA and miRNA) have been previously shown as potential promising targets for anti-cancer therapy development $[110,111,112,113]$. For instance, various strategies such as cell-based immunotherapy, antibody-based and drug-based therapies have been developed against EBV LMPs [111]. Cao and co-workers showed that DNAzyme resulted in significant tumour regression by targeting and cleaving off the LMP- mRNA from NPC patients [110]. Other tumour-promoting non-EBV proteins that could be targeted include EGFR $[114,115]$ and VEGF $[116,117]$. Similarly, targeting tumour-derived exosomes has been earmarked as a potent therapeutic strategy for cancer therapy although it must be noted that its potential remains to be explored [109,118].

In addition to carrying pathogenic factors that promote tumour formation and progression, tumour-derived exosomes could also play key roles in 
immune evasion of cancer cells as mentioned above $[24,25,34,38]$. Hence, blocking these exosomes may restore the activity of functional immune cells to kill the cancer cells. The immunosuppressive activity of NPC cell-derived and patient plasma-derived exosomes has been demonstrated on the PBMC-derived CD4+ $\mathrm{T}$ lymphocytes. The galectin-9-containing exosomes have inhibited the EBV-specific T-cell proliferation and induced apoptosis, hence preventing the cancer cells from T-cell-mediated recognition and killing of cancer cells [24,87]. Development of a therapeutic strategy to block the galectin-9 or other proteins from the exosomes may therefore restore the normal immune surveillance. Over the past few years, considerable work has also been done on targeting the whole exosomes rather than targeting the specific proteins $[119,120]$. The exosomal removal using a modified kidney dialysis system has also been proposed to be introduced in the clinical settings [119]. These findings suggest that diminishing or eliminating the tumorigenic exosomes may be a good therapeutic approach to reverse the exosome-mediated cancer progression, particularly in the aspect of immune dysregulation.

In addition to serving as therapeutic targets, exosomes have been interestingly adopted as vehicles for therapeutics delivery $[121,122,123]$. Ohno and coworkers demonstrated that the Let-7a miRNA-loaded exosomes targeting EGFR could be efficiently delivered to target the breast cancer-derived murine xenograft tissues [121]. Similarly, Qi and coworkers showed that exosome-based delivery of drug in murine hepatoma xenografts could cause significant regression of tumour size [122]. Hence, loading the existing exosomes with anti-EBV or anti-cancer therapeutic drugs or compounds might be a potent strategy to inhibit the tumour formation in addition to other advantages such as increasing the drug stability/ bioavailability and improving the drug delivery and cell permeation. Further investigations are warranted to examine the potency of these anti-EBV and/or anti-cancer exosomes in therapeutic intervention.

\section{Potential challenges and limitations}

While several lines of evidences showed that the EBV-containing exosomes can be targeted for the development of biomarker and/or anti-cancer therapy (Figure 2), several considerations need to be taken into account to ensure the success of the therapy and biomarker development. Sensitivity and specificity are important criteria for the cancer biomarker development. While the exosomecontaining EBV oncogenic contents (eg. EBNAs,
LMPs, and EBERs) have tumour-promoting and pathogenic properties and are expressed during the disease, they are not cancer type-specific and could be detected in various EBV-associated cancers such as NPC, GC, and BL [27]. Hence, other non-invasive tests such as cancer antigen screening and magnetic resonance imaging (MRI) are required in combination with potential exosomal analysis to enhance the specificity of diagnosis towards a particular cancer type. Furthermore, the expression of latent proteins LMPs such as LMP1 may be low or even at undetectable level especially during the early stage of tumour formation [124], hence the resultant sensitivity may be a potential issue. Comparatively, EBERs are more suitable targets for detection as they are consistently expressed at detectable amount in all EBV latently infected cells or tissues [44].

As an important therapeutic target, EBV proteins/nucleic acids can be targeted for tumour regression. There are a few challenges that may be encountered during the development of anti-cancer therapies. The exosomes play important roles in cell-cell communication mainly by regulating cellular processes, hence complete removal of exosomes is not a feasible therapeutic strategy as it will affect the well-being of cellular processes under a normal condition [30]. Hence, it is important to specifically target only the exosomes enriched with the pathogenic factors without affecting the biological activities of other existing exosomes. In addition, the exosomal contents may largely vary and heterogeneous depending on the sources or origins [12]. This may be another challenge especially the targeted exosomes are from the patient's circulating blood. Furthermore, some potential targets may be scarcely expressed or not at all in the targeted exosomes which will lead to the treatment failure. Other considerations in exploiting exosomes for drug delivery include the dosage of exosome-targeting drugs that may vary from one to another, the delivery system for targeting exosomes, the stability of the delivered therapeutic molecules, and the treatment course. Further works are needed to evaluate the clinical safety of exosome-targeting treatment strategy.

\section{Conclusions}

EBV-associated exosomes undoubtedly play pivotal roles in the pathogenesis of the viral infections and EBV-associated cancers especially in NPC. The cumulative findings suggest that the EBV-associated exosomes are ideal targets for the development of diagnostic biomarker and anticancer therapy. However, several issues need to be taken into account during the development as abovementioned. Since 
limited studies have been carried out in general, future investigations are warranted to evaluate the association of EBV-exosomes in other related cancers such as GC, BL, and NHL. The targeted exosomes could also be potentially developed into vaccines for EBV infections, hence diminishing the chances of developing the EBV-associated cancers. To our best understanding, this is the first review that discusses the role of pathogenic exosomes in contributing to EBV infection and the associated cancers in a much focused approach.

\section{Abbreviations}

AGO2: Argonaute 2; AIDS: acquired immune deficiency syndrome; BL: Burkitt lymphoma; DCs: dendritic cells; dUTPase: deoxyuridine triphosphatase; EBERs: Epstein-Barr virus-encoded small RNAs; EBNAs: Epstein-Barr virus nuclear antigens; EBV: Epstein-Barr virus; ERK: extracellular signal-regulated kinases; EGFR: epidermal growth factor receptor; EMT: epithelial-mesenchymal transition; EVs: extracellular vesicles; FGF: fibroblasts growth factor; GC: gastric carcinoma; $\mathrm{HCV}$ : hepatitis C virus; HIF1a: hypoxia-inducible factor 1a; HIV-I: human immunodeficiency virus type I; HL: Hodgkin lymphoma; HPV: human papilloma virus; HTLV-I: human T-cell lymphotropic virus type I; ICAM-1: intercellular adhesion molecule 1; ILVs: intraluminal vesicles; JAK: janus kinase; JNK: c-Jun $\mathrm{N}$ terminal kinase; La: lupus antigen; LCL: lymphoblastoid cell line; LMP-1: latency membrane protein 1; LMP-2A: latency membrane protein 2A; MAPK: mitogen-activated protein kinase; MCD: methyl-beta-cyclodextrin; MRI: magnetic resonance imaging; mRNAs: messenger RNAs; miRNAs: micro RNAs; MVBs: Multivesicular bodies; NFkB: nuclear factor kappa beta; NHL: Non Hodgkin lymphoma; NPC: nasopharyngeal carcinoma; PBMCs: peripheral blood mononuclear cells; PI3K: phosphoinositide 3-kinase; RISC: RNA-induced silencing complex; STAT: signal transducers and activators of transcription; TRBP: TAR RNA binding protein; VEGF: vascular endothelial growth factor.

\section{Acknowledgements}

The authors would like to thank the Director General of Health Malaysia for permission to publish this study and the Director of the Institute for Medical Research for her support. We would like to thank Sunway Internal Research Grant 2016 (INTM-2017-SIDS-SIHD-01) and Rewarding Research Output (RRO) (INT-RRO-2017-095) from Sunway University and National Cancer Council Malaysia (MAKNA) Cancer Research Award (CRA) 2016
(EXT-SIDS-SIHD-MAKNA-2017-01)

for

partly supporting this work.

\section{Competing Interests}

The authors have declared that no competing interest exists.

\section{References}

1. HogenEsch H, Nikitin AY. Challenges in pre-clinical testing of anti-cancer drugs in cell culture and in animal models. J Control Release. 2012;164:183-6.

2. Hait WN. Anticancer drug development: the grand challenges. Nat Rev Drug Discov. 2010;9:253-4

3. Thanki K, Gangwal RP, Sangamwar AT, et al. Oral delivery of anticancer drugs: challenges and opportunities. J Control Release. 2013;170:15-40.

4. Stuurman FE, Nuijen B, Beijnen JH, et al. Oral anticancer drugs: mechanisms of low bioavailability and strategies for improvement. Clin Pharmacokinet. 2013;52:399-414.

5. Chen F, Zhuang $X$, Lin L, et al. New horizons in tumour microenvironment biology: challenges and opportunities. BMC Med. 2015;13:45.

6. Sun Y. Tumour microenvironment and cancer therapy resistance. Cancer Lett. 2016;380:205-15.

7. Woller N, Kühnel F. Virus infection, inflammation and prevention of cancer. Recent Results Cancer Res. 2014;193:33-58.

8. Chiantore MV, Mangino G, Zangrillo MS, et al. Role of the microenvironment in tumorigenesis : focus on virus-induced tumours. Curr Med Chem. 2015;22:958-74.

9. Kahlert C, Kalluri R. Exosomes in tumour microenvironment influence cancer progression and metastasis. J Mol Med (Berl). 2013; 91:431-7.

10. Roma-Rodrigues C, Fernandes AR, Baptista PV. Exosome in tumour microenvironment: overview of the crosstalk between normal and cancer cells. Biomed Res Int. 2014;2014:179486.

11. Milane L, Singh A, Mattheolabakis G, et al. Exosome mediated communication within the tumour microenvironment. J Control Release. 2015;219:278-94.

12. Teow SY, Nordin AC, Ali SA, et al. Exosomes in human immunodeficiency virus type I pathogenesis: threat or opportunity? Adv Virol. 2016;2016:9852494

13. Madison MN, Okeoma CM. Exosomes: implications in HIV-1 pathogenesis. Viruses. 2015;7:4093-118.

14. Schwab A, Meyering SS, Lepene B, et al. Extracellular vesicles from infected cells: potential for direct pathogenesis. Front Microbiol. 2015;6:1132.

15. Alenquer M, Amorim MJ. Exosome biogenesis, regulation, and function in viral infection. Viruses. 2015;7:5066-83.

16. Kowal J, Arras G, Colombo M, et al. Proteomic comparison defines novel markers to characterize heterogeneous populations of extracellular vesicle subtypes. Proc Natl Acad Sci U S A. 2016;113:E968-77.

17. Iwakiri D. Epstein-Barr virus-encoded RNAs: key molecules in viral pathogenesis. Cancers. 2014;6:1615-30.

18. Canitano A, Venturi $G$, Borghi $M$, et al. Exosomes released in vitro from Epstein-Barr virus (EBV)-infected cells contain EBV-encoded latent phase mRNAs. Cancer Lett. 2013;337:193-9.

19. Longatti $\mathrm{A}$. The dual role of exosomes in hepatitis $\mathrm{A}$ and $\mathrm{C}$ virus transmission and viral immune activation. Viruses. 2015;7:6707-15.

20. Chahar HS, Bao X, Casola A. Exosomes and their role in the life cycle and pathogenesis of RNA viruses. Viruses. 2015;7:3204-25.

21. Jaworski E, Narayanan A, Van Duyne R, et al. Human T-lymphotropic virus type 1-infected cells secrete exosomes that contain Tax protein. J Biol Chem. 2014;289:22284-305.

22. Meckes DG Jr. Exosomal communication goes viral. J Virol. 2015;89:5200-3.

23. Aga M, Bentz GL, Raffa S, et al. Exosomal HIF1a supports invasive potential of nasopharyngeal carcinoma-associated LMP--positive exosomes. Oncogene. 2014;33:4613-22.

24. Keryer-Bibens C, Pioche-Durieu C, Villemant C, et al. Exosomes released by EBV-infected nasopharyngeal carcinoma cells convey the viral latent membrane protein 1 and the immunomodulatory protein galectin 9. BMC Cancer. 2006;6:283.

25. Mrizak D, Martin N, Barjon C, et al. Effect of nasopharyngeal carcinoma-derived exosomes on human regulatory $\mathrm{T}$ cells. J Natl Cancer Inst. 2014;107:363.

26. Liu $J$, Sun $H$, Wang $X$, et al. Increased exosomal microRNA-21 and microRNA-146a levels in the cervicovaginal lavage specimens of patients with cervical cancer. Int J Mol Sci. 2014;15:758-73.

27. Ko YH. EBV and human cancer. Exp Mol Med. 2015;47:e130.

28. Pattle SB, Farrell PJ. The role of Epstein-Barr virus in cancer. Expert Opin Biol Ther. 2006;6:1193-205.

29. Yang C, Robbins PD. The roles of tumour-derived exosomes in cancer pathogenesis. Clin Dev Immunol. 2011;2011:842849.

30. Zhang X, Yuan X, Shi H, et al. Exosomes in cancer: small particle, big player. J Hematol Oncol. 2015;8:83. 
31. Meckes DG, Shair KHY, Marquitz AR, et al. Human tumour virus utilizes exosomes for intercellular communication. Proc Natl Acad Sci USA. 2010;107:20370-5.

32. Thompson MP, Kurzrock R. Epstein-Barr virus and cancer. Clin Cancer Res. 2004;10:803-21.

33. Young LS, Yap LF, Murray PG. Epstein-Barr virus: more than 50 years old and still providing surprises. Nat Rev Cancer. 2016 ;16:789-802.

34. Ahmed W, Philip PS, Tariq S, et al. Epstein-Barr virus-encoded small RNAs (EBERs) are present in fractions related to exosomes released by EBV-transformed cells. PLoS One. 2014;9:e99163.

35. Ahsan N, Kanda T, Nagashima K, et al. Epstein-Barr virus transforming protein LMP- plays a critical role in virus production. J Virol. 2005;79:4415-24.

36. Hurwitz SN, Nkosi D, Conlon MM, et al. CD63 regulates Epstein-Barr virus LMP- exosomal packaging, enhancement of vesicle production, and non-canonical NF-kB signaling. J Virol. 2016:02251-16.

37. Nanbo A, Kawanishi E, Yoshida R, et al. Exosomes derived from Epstein-Barr virus-infected cells are internalized via caveola-dependent endocytosis and promote phenotypic modulation in target cells. J Virol. 2013;87:10334-47.

38. Flanagan J, Middeldorp J, Sculley T. Localization of the Epstein-Barr virus protein LMP 1 to exosomes. J Gen Virol. 2003;84:1871-9.

39. Meckes DG Jr, Gunawardena HP, Dekroon RM, et al. Modulation of B-cell exosome proteins by gamma herpesvirus infection. Proc Natl Acad Sci USA. 2013;110:E2925-33

40. Ikeda M, Longnecker R. Cholesterol is critical for Epstein-Barr virus latent membrane protein 2A trafficking and protein stability. Virology. 2007;360:461-8.

41. Allen MD, Young LS, Dawson CW. The Epstein-Barr virus-encoded LMP-2A and LMP2B proteins promote epithelial cell spreading and motility. J Virol. 2005;79:1789-802.

42. Rechsteiner MP, Berger C, Zauner L, et al. Latent membrane protein 2B regulates susceptibility to induction of lytic Epstein-Barr virus infection. J Virol. 2008;82:1739-47.

43. Ahmed W, Khan G. The labyrinth of interactions of Epstein-Barr virus-encoded small RNAs. Rev Med Virol. 2014;24: 3-14.

44. Iwakiri D, Takada K. Role of EBERs in the pathogenesis of EBV infection. Adv Cancer Res. 2010;107:119-36.

45. Pegtel DM, Cosmopoulos K, Thorley-Lawson DA, et al. Functional delivery of viral miRNAs via exosomes. Proc Natl Acad Sci USA. 2010;107:6328-33.

46. Verweij FJ, van Eijndhoven MAJ, Middeldorp J, et al. Analysis of viral microRNA exchange via exosomes in vitro and in vivo. Methods Mol Biol. 2013;1024:53-68.

47. Vallhov H, Gutzeit C, Johansson SM, et al. Exosomes containing glycoprotein 350 released by EBV-transformed B cells selectively target B cells through CD21 and block EBV infection in vitro. J Immunol. 2011;186:73-82.

48. Dukers DF, Meij P, Vervoort MB, et al. Direct immunosuppressive effects of EBV-encoded latent membrane protein 1. J Immunol. 2000;165:663-70.

49. Ceccarelli S, Visco V, Raffa S, et al. Epstein-Barr virus latent membrane protein 1 promotes concentration in multivesicular bodies of fibroblast growth factor 2 and its release through exosomes. Int J Cancer. 2007;121:1494-506.

50. Busson P, Zhang Q, Guillon JM, et al. Elevated expression of ICAM1 (CD54) and minimal expression of LFA3 (CD58) in Epstein-Barr-virus-positive nasopharyngeal carcinoma cells. Int J Cancer. 1992;50:863-7.

51. Jung WC, Jang YJ, Kim JH, et al. Expression of intercellular adhesion molecule-1 and e-selectin in gastric cancer and their clinical significance. J Gastric Cancer. 2012;12:140-8

52. Terol MJ, López-Guillermo A, Bosch F, et al. Expression of the adhesion molecule ICAM-1 in non-Hodgkin's lymphoma: relationship with tumour dissemination and prognostic importance. J Clin Oncol. 1998;16:35-40.

53. Verweij FJ, van Eijndhoven MA, Hopmans ES, et al. LMP- association with $\mathrm{CD} 63$ in endosomes and secretion via exosomes limits constitutive NF-KB activation. EMBO J. 2011;30:2115-29.

54. Thorley-Lawson DA, Gross A. Persistence of the Epstein-Barr virus and the origins of associated lymphomas. N Engl J Med. 2004;350:1328-37.

55. Eliopoulos AG, Young LS. Activation of the cJun N-terminal kinase (JNK) pathway by the Epstein-Barr virus-encoded latent membrane protein 1 (LMP-). Oncogene. 1998;16:1731-42.

56. Thornburg NJ, Kulwichit W, Edwards RH, et al. LMP- signaling and activation of NF-kappaB in LMP- transgenic mice. Oncogene. 2006;25:288-97.

57. Johansson $\mathrm{P}$, Jansson A, Rüetschi $\mathrm{U}$, et al. The p38 signaling pathway upregulates expression of the Epstein-Barr virus LMP- oncogene. J Virol. 2010;84:2787-97.

58. Lambert SL, Martinez OM. Latent membrane protein 1 of EBV activates phosphatidylinositol 3-kinase to induce production of IL-10. J Immunol. 2007;179:8225-34.

59. Dawson CW, Laverick L, Morris MA, et al. Epstein-Barr virus-encoded LMPregulates epithelial cell motility and invasion via the ERK-MAPK pathway. J Virol. 2008;82:3654-64.

60. Chen H, Hutt-Fletcher L, Cao L, et al. A positive autoregulatory loop of LMPexpression and STAT activation in epithelial cells latently infected with Epstein-Barr virus. J Virol. 2003;77:4139-48.

61. Husaini R, Ahmad M, Khoo AS. Epstein-Barr virus latent membrane protein LMP1 reduces p53 protein levels independent of the PI3K-Akt pathway. BMC Res Notes. 2011;4:551.

62. Hoe S, Lee E, Khoo AS, Peh S. p53 and nasopharyngeal carcinoma: a Malaysian study. Pathology. 2009;41:561-5.
63. Kung CP, Meckes DG Jr, Raab-Traub N. Epstein-Barr virus LMP- activates EGFR, STAT3, and ERK through effects on PKCdelta. J Virol. 2011;85:4399-408.

64. Stewart S, Dawson CW, Takada K, et al. Epstein-Barr virus-encoded LMP-2A regulates viral and cellular gene expression by modulation of the NF-kappaB transcription factor pathway. Proc Natl Acad Sci USA. 2004;101:15730-5.

65. Pang MF, Lin KW, Peh SC. The signaling pathways of Epstein-Barr virus-encoded latent membrane protein 2A (LMP-2A) in latency and cancer. Cell Mol Biol Lett. 2009;14:222-47.

66. Shair KH, Bendt KM, Edwards RH, et al. Epstein-Barr virus-encoded latent membrane protein 1 (LMP-) and LMP-2A function cooperatively to promote carcinoma development in a mouse carcinogenesis model. J Virol. 2012;86:5352-65.

67. Dawson CW, Port RJ, Young LS. The role of the EBV-encoded latent membrane proteins LMP- and LMP2 in the pathogenesis of nasopharyngeal carcinoma (NPC). Semin Cancer Biol. 2012;22:144-53.

68. Shinozaki-Ushiku A, Kunita A, Fukayama M. Update on Epstein-Barr virus and gastric cancer (review). Int J Oncol. 2015;46:1421-34.

69. Brooks L, Yao QY, Rickinson AB, et al. Epstein-Barr virus latent gene transcription in nasopharyngeal carcinoma cells: coexpression of EBNA1, LMP-, and LMP2 transcripts. J Virol. 1992;66:2689-97.

70. He L, Hannon GJ. MicroRNAs: small RNAs with a big role in gene regulation. Nat Rev Genet. 2004;5:522-31.

71. Bartel DP. MicroRNA target recognition and regulatory functions. Cell. 2009;136:215-33.

72. Lo AK, To KF, Lo KW, et al. Modulation of LMP- protein expression by EBV-encoded microRNAs. Proc Natl Acad Sci USA. 2007;104:16164-9.

73. Zhu JY, Pfuhl T, Motsch N, et al. Identification of novel Epstein-Barr virus microRNA genes from nasopharyngeal carcinomas. J Virol. 2009;83:3333-41.

74. Cai L, Ye Y, Jiang Q, et al. Epstein-Barr virus-encoded microRNA BART1 induces tumour metastasis by regulating PTEN-dependent pathways in nasopharyngeal carcinoma. Nat Commun. 2015;6:7353.

75. Marquitz AR, Mathur A, Chugh PE, et al. Expression profile of microRNAs in Epstein-Barr virus-infected AGS gastric carcinoma cells. J Virol. 2014;88:1389-93.

76. Shinozaki-Ushiku A, Kunita A, Isogai M, et al. Profiling of virus-encoded microRNAs in Epstein-Barr virus-associated gastric carcinoma and their roles in gastric carcinogenesis. J Virol. 2015;89:5581-91.

77. Piccaluga PP, Navari M, De Falco G, et al. Virus-encoded microRNA contributes to the molecular profile of EBV-positive Burkitt lymphomas. Oncotarget. 2016;7:224-40.

78. Vereide DT, Seto E, Chiu YF, et al. Epstein-Barr virus maintains lymphomas via its miRNAs. Oncogene. 2014;33:1258-64.

79. Pandya D, Mariani M, He S, et al. Epstein-Barr Virus microRNA expression increases aggressiveness of solid malignancies. PLoS One. 2015;10:e136058.

80. Melo SA, Sugimoto H, O'Connell JT, et al. Cancer exosomes perform cell-independent microRNA biogenesis and promote tumorigenesis. Cancer Cell. 2014;26:707-21.

81. Thind A, Wilson C. Exosomal miRNAs as cancer biomarkers and therapeutic targets. J Extracell Vesicles. 2016;5:31292.

82. Ye SB, Li ZL, Luo DH, et al. Tumour-derived exosomes promote tumour progression and T-cell dysfunction through the regulation of enriched exosomal microRNAs in human nasopharyngeal carcinoma. Oncotarget. 2014;5:5439-52.

83. Yang Q, Diamond MP, Al-Hendy A. The emerging role of extracellular vesicle-derived miRNAs: implication in cancer progression and stem cell related diseases. J Clin Epigenet. 2016;2:pii:13.

84. Matsumura T, Sugimachi $K$, Iinuma $H$, et al. Exosomal microRNA in serum is a novel biomarker of recurrence in human colorectal cancer. $\mathrm{Br} \mathrm{J}$ Cancer 2015:113:275-81.

85. Choi H, Lee H, Kim SR, et al. Epstein-Barr virus-encoded microRNA BART15-3p promotes cell apoptosis partially by targeting BRUCE. J Virol. 2013:87:8135-44.

86. Klibi J, Niki T, Riedel A, et al. Blood diffusion and Th1-suppressive effects of galectin-9-containing exosomes released by Epstein-Barr virus-infected nasopharyngeal carcinoma cells. Blood. 2009;113:1957-66.

87. Daker M, Bhuvanendran S, Ahmad M, Takada K, Khoo AS. Deregulation of lipid metabolism pathway genes in nasopharyngeal carcinoma cells. Mol Med Rep. 2013;7:731-41.

88. Ariza ME, Rivailler P, Glaser $R$, et al. Epstein-Barr virus encoded dUTPase containing exosomes modulate innate and adaptive immune responses in human dendritic cells and peripheral blood mononuclear cells. PLoS One 2013;8:e69827.

89. Huang $\mathrm{SH}, \mathrm{Li} \mathrm{Y}$, Zhang J, et al. Epidermal growth factor receptor-containing exosomes induce tumour-specific regulatory $\mathrm{T}$ cells. Cancer Invest. 2013;31:330-5

90. Ansari MA, Singh VV, Dutta S, et al. Constitutive interferon-inducible protein 16-inflammasome activation during Epstein-Barr virus latency I, II, and III in B and epithelial cells. J. Virol. 2013;87: 8606-23.

91. Shao H, Chung J, Issadore D. Diagnostic technologies for circulating tumour cells and exosomes. Biosci Rep. 2016;36:e00292.

92. Lin J, Li J, Huang B, et al. Exosomes: novel biomarkers for clinical diagnosis. Sci World J. 2015;2015:657086.

93. Munson P, Shukla A. Exosomes: potential in cancer diagnosis and therapy. Medicines (Basel). 2015;2:310-27. 
94. An T, Qin S, Xu Y, et al. Exosomes serve as tumour markers for personalized diagnostics owing to their important role in cancer metastasis. J Extracell Vesicles. 2015;4:27522.

95. Cappello F, Logozzi M, Campanella C, et al. Exosome levels in human body fluids: A tumour marker by themselves? Eur J Pharm Sci. 2016;96:93-8.

96. Houali K, Wang X, Shimizu Y, et al. A new diagnostic marker for secreted Epstein-Barr virus encoded LMP- and BARF1 oncoproteins in the serum and saliva of patients with nasopharyngeal carcinoma. Clin Cancer Res. 2007;13:4993-5000.

97. Mao $\mathrm{Y}$, Zhang DW, Zhu H, et al. LMP- and LMP-2A are potential prognostic markers of extranodal NK/T-cell lymphoma, nasal type (ENKTL). Diagn Pathol. 2012;7:178

98. Yip TT, Ngan RK, Fong AH, et al. Application of circulating plasma/serum EBV DNA in the clinical management of nasopharyngeal carcinoma. Oral Oncol. 2014;50:527-38

99. De Paoli P, Pratesi C, Bortolin MT. The Epstein Barr virus DNA levels as a tumour marker in EBV-associated cancers. J Cancer Res Clin Oncol. 2007;133: $809-15$.

100. Stevens SJ, Verkuijlen SA, Hariwiyanto B, et al. Noninvasive diagnosis of nasopharyngeal carcinoma: nasopharyngeal brushings reveal high Epstein-Barr virus DNA load and carcinoma-specific viral BARF1 mRNA. Int J Cancer. 2006;119:608-14.

101. Stevens SJC, Verkuijlen SAWM, Hariwiyanto B, et al. Diagnostic value of measuring Epstein-Barr Virus (EBV) DNA load and carcinoma-specific viral mRNA in relation to anti-EBV immunoglobulin A (IgA) and IgG antibody levels in blood of nasopharyngeal carcinoma patients from Indonesia. J Clin Microbiol. 2005;43:3066-73.

102. Zhang G, Zong J, Lin S, et al. Circulating Epstein-Barr virus microRNAs miR-BART7 and miR-BART13 as biomarkers for nasopharyngeal carcinoma diagnosis and treatment. Int J Cancer. 2015;136:E301-12.

103. Zheng $\mathrm{XH}$, Lu LX, Cui $\mathrm{C}$, et al. Epstein-Barr virus mir-bart1-5p detection via nasopharyngeal brush sampling is effective for diagnosing nasopharyngeal carcinoma. Oncotarget. 2016;7:4972-80.

104. Gourzones C, Ferrand FR, Vérillaud B, et al. Biological tools for NPC population screening and disease monitoring. In: Busson $\mathrm{P}$, ed. Nasopharyngeal Carcinoma: Keys for Translational Medicine and Biology. Austin and New-York: Landes and Springer; 2012: 101-17.

105. Dales JP, Beaufils N, Silvy M, et al. Hypoxia inducible factor 1a gene (HIF-1a) splice variants: potential prognostic biomarkers in breast cancer. BMC Med. 2010;8:44.

106. Weber DC, Tille JC, Combescure $C$, et al. The prognostic value of expression of HIF1a, EGFR and VEGF-A, in localized prostate cancer for intermediate- and high-risk patients treated with radiation therapy with or without androgen deprivation therapy. Radiat Oncol. 2012;7:66.

107. Valadi $\mathrm{H}$, Ekström $\mathrm{K}$, Bossios A, et al. Exosome-mediated transfer of mRNAs and microRNAs is a novel mechanism of genetic exchange between cells. Nat Cell Biol. 2007;9:654-9.

108. Iero M, Valenti R, Huber V, et al. Tumour-released exosomes and their implications in cancer immunity. Cell Death Differ. 2008;15:80-8.

109. Yu S, Cao H, Shen B, et al. Tumour-derived exosomes in cancer progression and treatment failure. Oncotarget. 2015;6:37151-68.

110. Cao Y, Yang L, Jiang W, et al. Therapeutic evaluation of Epstein-Barr virus-encoded latent membrane protein-1 targeted DNAzyme for treating of nasopharyngeal carcinomas. Mol Ther. 2014;22:371-7.

111. Hutajulu SH, Kurnianda J, Tan IB, et al. Therapeutic implications of Epstein-Barr virus infection for the treatment of nasopharyngeal carcinoma. Ther Clin Risk Manag. 2014;10:721-36.

112. Lee HG, Kim H, Kim EJ, et al. Targeted therapy for Epstein-Barr virus-associated gastric carcinoma using low-dose gemcitabine-induced lytic activation. Oncotarget. 2015;6:31018-29.

113. Stoker SD, Novalić Z, Wildeman MA, et al. Epstein-Barr virus-targeted therapy in nasopharyngeal carcinoma. J Cancer Res Clin Oncol. 2015;141:1845-57

114. Chua DT, Wei WI, Wong MP, et al. Phase II study of gefitinib for the treatment of recurrent and metastatic nasopharyngeal carcinoma. Head Neck. 2008;30:863-7.

115. Ma B, Hui EP, King A, et al. A phase II study of patients with metastatic or locoregionally recurrent nasopharyngeal carcinoma and evaluation of plasma Epstein-Barr virus DNA as a biomarker of efficacy. Cancer Chemother Pharmacol. 2008;62:59-64.

116. Lee NY, Zhang Q, Pfister DG, et al. Addition of bevacizumab to standard chemoradiation for locoregionally advanced nasopharyngeal carcinoma (RTOG 0615): a phase 2 multi-institutional trial. Lancet Oncol. 2012;13:172-80.

117. Elser C, Siu LL, Winquist E, et al. Phase II trial of sorafenib in patients with recurrent or metastatic squamous cell carcinoma of the head and neck or nasopharyngeal carcinoma. J Clin Oncol.2007;25:3766-73.

118. De Toro J, Herschlik L, Waldner C, et al. Emerging roles of exosomes in normal and pathological conditions: new insights for diagnosis and therapeutic applications. Front Immunol. 2015;6:203.

119. Marleau AM, Chen CS, Joyce JA, et al. Exosome removal as a therapeutic adjuvant in cancer. J Transl Med. 2012;10:134.

120. Tickner JA, Urquhart AJ, Stephenson SA, et al. Functions and therapeutic roles of exosomes in cancer. Front Oncol. 2014;4:127.
121. Ohno S, Takanashi M, Sudo K, et al. Systemically injected exosomes targeted to EGFR deliver antitumour microRNA to breast cancer cells. Mol Ther. 2013;21:185-91.

122. Qi H, Liu C, Long $\mathrm{L}$, et al. Blood exosomes endowed with magnetic and targeting properties for cancer therapy. ACS Nano. 2016;10:3323-33.

123. Johnsen KB, Gudbergsson JM, Skov MN, et al. A comprehensive overview of exosomes as drug delivery vehicles - endogenous nanocarriers for targeted cancer therapy. Biochim Biophys Acta. 2014;1846:75-87.

124. Hannigan A, Wilson JB. Evaluation of LMP- of Epstein-Barr virus as a therapeutic target by its inhibition. Mol Cancer. 2010;9:184. 